\title{
MODELE EN COUCHES ET MOUVEMENTS COLLECTIFS
}

\author{
Par J. YOCCOZ, \\ Laboratoire de Physique et Chimie Nucléaires du Collège de France.
}

\begin{abstract}
Sommaire. - Une méthode variationelle, proposée par Hill et Wheeler, est utilisée pour décrire les mouvements collectifs du noyau. Les résultats, en ce qui conserme la relation entre le moment d'inertie I du noyau et sa déformation, sont meilleurs que ceux obtenus avec le modèle hydrodynamique.
\end{abstract}

\begin{abstract}
A variational method, first proposed by Hill and Wheeler, is used for the description of the collective motions of the nuclei. The results, for the relation between the moment of inertia and the deformation, are better than those obtained with hydrodynamic model.
\end{abstract}

Le modèle en couches classique suppose l'existence d'un puits de pötentiel équivalent en première approximation à l'ensemble des interactions entre particules. Nous nous proposons d'utiliser un certain nombre de paramètres caractéristiques de ce puits relatifs à la position de son centre, son orientation dans l'espace s'il n'est pas sphérique, etc, pour décrire un mouvement collectif du noyau, l'idée physique étant que si nous entrâ̂nons le puits dans un certain mouvement, les nucléons seront entraînés dans ce même mouvement, au moins en. première approximation. On substituera donc à la fonction d'onde bâtie a sur la base du modèle en couches, ces paramètres étant fixés, une fonction d'onde décrivant aussi le mouvement collectif.

Par la suite, $a$ désignera symboliquement un (ou un ensemble) de ces paramètres. La fonction d'onde du modèle en couches, a étant fixé, s'écrira $\psi\left(\overrightarrow{r_{1}}, \overrightarrow{r_{2}} \ldots \overrightarrow{r_{\mathrm{A}}}, a\right)$. La fonction d'onde décrivant le noyau sera

$$
-\bar{\Psi}-\left(\overrightarrow{r_{1}} \ldots \overrightarrow{r_{\mathrm{A}}}\right)=\int X(\mathrm{a}) \psi\left(\overrightarrow{r_{1}} \ldots \overrightarrow{r_{\mathrm{A}}}, a\right) \mathrm{d} a .
$$

$X(a)$ étant déterminé par une méthode variationnelle, et devant en outre satisfaire certaines conditions supplémentaires appropriées. On obtient pour $X(a)$ une équation intégrale

$$
\int X(a)\left[H\left(a^{\prime}, a\right)-E N\left(a^{\prime}, a\right)\right] \mathrm{d} a=0,
$$

quel que soit $a^{\prime}$, avec

$$
\begin{aligned}
& H\left(a^{\prime}, a\right)=\int \psi^{*}\left(\overrightarrow{r_{1}} \ldots \overrightarrow{r_{\mathrm{A}}} a^{\prime}\right) H \psi\left(\overrightarrow{r_{1}} \ldots \overrightarrow{r_{\mathrm{A}}} a\right) \mathrm{d} \overrightarrow{r_{1}} \ldots \mathrm{d} \overrightarrow{r_{\mathrm{A}}} \\
& N\left(a^{\prime}, a\right)=\int \psi^{*}\left(\vec { r _ { 1 } } \ldots \vec { r _ { \mathrm { A } } } a ^ { \prime } \left(\psi\left(\overrightarrow{r_{1}} \ldots \overrightarrow{r_{\mathrm{A}}} a\right) \mathrm{d} \overrightarrow{r_{1}} \ldots \mathrm{d} \overrightarrow{r_{\mathrm{A}}}\right.\right.
\end{aligned}
$$

Cette équation intégrale peut être résolue exactement dans le cas où $H\left(a^{\prime}, a\right)$ et $N\left(a^{\prime}, a\right)$ ne dépendent que de $a-a^{\prime}$ (mouvement de translation et de rotation), et il est possible d'en trouver une solution approximative, dans le cas des mouvements de vibration par une méthode basée essentiellement sur le fait que $H\left(a^{\prime}, a\right)$ et $N\left(a^{\prime}, a\right)$ présentent pour $a^{\prime}=a$ un maximum très aigu.

Il est certain que sauf les cas spéciaux où la fonction se met sous le forme $F(a) G\left(\overrightarrow{r_{1}} \ldots \overrightarrow{r_{\Delta}}\right)$, a fonction d'onde $\psi$ contient un certain couplagel entre mouvement interne et mouvement collectif. Si $\psi\left(\overrightarrow{r_{1}} \ldots \overrightarrow{r_{\mathrm{A}}}, a\right)$ décrit l'état fondamental du modèle en couches, et si l'énergie du mouvement collectif n'est pas assez grande pour exciter un état de mouvement interne, ce couplage aura des conséquences réduites. Par contre, la méthode peut devenir très mauvaise si ces conditions ne sont pas remplies.

En particulier, le mouvement du centre de gravité étant décrit par le mouvement du centre $R$ du puits, on trouve $X(\vec{R})=\mathrm{e}^{i \overrightarrow{h_{0}} \vec{R}}$, avec la condition $E=h\left(h_{0}\right) / n\left(\overrightarrow{h_{0}}\right)$, dans laquelle $h\left(\overrightarrow{h_{0}}\right)$ et $n\left(\vec{h}_{0}\right)$ sont les transformées de Fourrier des intégrales $H(\vec{p})$ et $N(\vec{\rho})$, avec $\vec{\rho}=\overrightarrow{R^{\prime}}-\vec{R}$. Pour donner un sens physique à $\overrightarrow{h_{0}}$, nous calculerons la valeur moyenne de l'opérateur $\vec{P}$ d'impulsion totale $\overrightarrow{\vec{P}}=\left\langle\Psi\left|\overrightarrow{P_{\mathrm{op}}}\right| \Psi\right\rangle$. Éliminant $\overrightarrow{\boldsymbol{h}_{0}}$ entre cette expression et la valeur de l'énergie exprimée précédemment, nous contrôlerons la validité de notre approximation en comparant, la valeur calculée $E=f \overline{(\vec{P})}$ et la valeur correcte $E=E_{0}+\frac{\overline{|\vec{P}|^{2}}}{2 \pi}$. Les résultats sont corrects si le modèle en couches est basé sur un potentiel harmonique, et pour la configuration décrivant l'état fondamental. Pour ce mềme potentiel, les résultats sont plus mauvais dans le cas de configurations décrivant des états excités au sens du modèle. Ils restent acceptables si cette excitation est faible et si le nombre atomique $A$ est grand.

Le mouvement de vibration (de volume ou de surface) peut aussi être décrit par cette méthode. 
Pour un nombre de nucléons suffisamment, grand, l'équation intégrale peut être remplacée par une équation différentielle, dont les solutions sont soit du type oscillateur harmonique, soit du type fonctions sphéroïdes suivant les approximations permises par la valeur plus ou moins grande de la compressibilité du noyau (oscillation de volume) ou de sa résistance à la déformation (oscillation de surface).

Nous considérerons enfin le mouvement de rotation dans le cas simple d'un noyau pair-pair, admettant un axe de symétrie, dans une configuration interne telle que la composante du moment angulaire le long de cet axe est nulle, et une rotation possible autour d'un axe perpendiculaire à l'axe de symétrie, décrite par un angle $\varphi$.

Avec une valeur $\rho=1,210^{-13} \mathrm{~cm}$ du rayon élémentaire nucléaire, et une valeur correcte du moment quadrupolaire intrinsèque, on trouve les valeurs suivantes pour la différence d'énergie $\varepsilon$ (en $\mathrm{keV}$ ) entre les états $J=2$ et $J=0$ :

\begin{tabular}{|c|c|c|c|}
\hline Noyau & ${ }_{90}^{60} \mathrm{Nd}^{150}$ & ${ }_{92}^{62} \mathrm{Sm}^{154}$ & ${ }_{114}^{76} \mathrm{Os}^{190}$ \\
\hline$\varepsilon$ calculé & 110 & 100 & 180 \\
\hline$\varepsilon$ expérimental & 230 & 82 & 135 \\
\hline
\end{tabular}

la valeur expérimentale pour ${ }^{76} \mathrm{Os}{ }^{190}$ étant extrapolée par rapport aux valeurs trouvées pour $\mathrm{Os}^{186},{ }^{188}$. Les écarts sont beaucoup plus grands si les moments d'inertie sont calculés sur la base d'un modèle hydrodynamique, puisqu'ils sont de l'ordre de 4. Étant donnée la grossièreté du modèle, il semble que notre méthode soit susceptible de décrire correctement le mouvement de rotation, et de donner une relation acceptable entre $Q_{0}$ et $I$. 